\title{
LINEAR OPERATORS ON POLYNOMIALS PRESERVING ROOTS IN OPEN CIRCULAR DOMAINS
}

\author{
EUGENY MELAMUD
}

\begin{abstract}
In the present paper we answer a question raised by J. Borcea and P. Brändén and give a description of the class of operators preserving roots in open circular domains, i.e., in images of the open upper half-plane under the Möbius transformations. Our second result is a description of the difference between $\mathcal{A}(G)$ (the class of operators preserving roots in an open set $G$ ) and $\mathcal{A}(\bar{G})$ (the class of operators preserving roots in $\bar{G}$ ).
\end{abstract}

\section{INTRODUCTION}

Let $K$ be an arbitrary set in the complex plane, by $\pi(K)$ we denote the set of all polynomials with roots in $K$ and by $\pi_{n}(K)$ we denote the set of polynomials from $\pi(K)$ of degree at most $n$. As usual, we denote by $\mathbb{C}[z]$ the set of all polynomials with complex coefficients. We say that a linear operator $T$ belongs to the class $\mathcal{A}(K)$, if for any $p \in \pi(K)$ the polynomial $T p$ belongs to $\pi(K) \cup\{0\}$. This paper is concerned with the problem of description of the class $\mathcal{A}(K)$.

Let us point out that we do not assume operators from $\mathcal{A}(K)$ to be continuous. We will need continuity in several places, but in each case operator will act on a finite-dimensional subspace of $\mathbb{C}[z]$, hence $T$ will be continuous on that subspace.

Despite of a long history of the problem, it is completely solved only for closed circular domains (half-plane and disk) and their boundaries (line and circle). Probably the first widely known result concerning linear operators and zero distribution, is the following Pólya-Schur theorem, describing the so called multiplier sequences [5].

Theorem 1.1. (Pólya-Schur, 5]) Let $T$ be a diagonal operator corresponding to a real sequence $\left\{a_{k}\right\}_{k=0}^{\infty}$ given by $T\left[z^{k}\right]=a_{k} z^{k}$. Then the following are equivalent:

(1) The operator $T$ preserves polynomials with real zeros;

(2) For any positive integer $n$ the polynomials

$$
T\left[(1+z)^{n}\right]
$$

belong to the Laguerre-Pólya class $\mathcal{L P}\left(\mathbb{R}_{+}\right) \cup \mathcal{L P}\left(\mathbb{R}_{-}\right)$;

(3) The series

$$
T\left[e^{z}\right]=\sum_{k=0}^{\infty} \frac{c_{k}}{n !} z^{k}
$$

converges in the whole plane and belongs to the class $\mathcal{L P}\left(\mathbb{R}_{+}\right) \cup \mathcal{L P}\left(\mathbb{R}_{-}\right)$.

1991 Mathematics Subject Classification. Primary 30C15; Secondary 32A60, 47B38.

Linear operators on polynomial spaces, zeros of polynomials, circular domains, Pólya-Schur theorem. 
The mentioned classes $\mathcal{L P}\left(\mathbb{R}_{+}\right)$and $\mathcal{L P}\left(\mathbb{R}_{-}\right)$play an important role in the theory of entire functions; they consist of entire functions which are limits, uniformly on compact sets, of polynomials with zeros respectively in $\mathbb{R}_{+}=[0, \infty)$ or in $\mathbb{R}_{-}=(-\infty, 0]$. These classes are a key object in various classifications of operators preserving roots on the real axis.

Later a similar result was obtained for operators commuting with differentiation operator $D\left[2\right.$ and for operators commuting with inverted differentiation $z^{2} D[1$. Still the description of operators which preserve roots on the real axis or in the half-plane and do not satisfy some special conditions was an open problem till recently. A classification of linear operators preserving roots in circular domains and on their boundaries obtained recently by J. Borcea and P. Brändén [4] was a real breakthrough. To introduce their result we will need the following definition.

Definition 1.1. Let $K$ be an arbitrary set in the complex plane. A polynomial $p \in \mathbb{C}\left[z_{1}, \ldots, z_{n}\right]$ is said to be $K$-stable, if $p\left(\zeta_{1}, \ldots, \zeta_{n}\right) \neq 0$ whenever $\zeta_{i} \in K$, $1 \leq i \leq n$.

Denote by $\Phi(z)$ the Möbius transformation given by

$$
\Phi(z)=\frac{a z+b}{c z+d}, \quad \text { where } \quad a d-b c \neq 0 .
$$

For a linear operator $T: \mathbb{C}[z] \rightarrow \mathbb{C}[z]$, let us extend it to the set of bivariate polynomials $\mathbb{C}[z, w]$ as if $w$ is a constant, by putting $T\left[z^{k} w^{m}\right]=w^{m} T\left[z^{k}\right]$.

Denote by $H$ the upper half-plane $\{z: \Im z>0\}$ and put $C=\Phi^{-1}(H)$. The following theorem [4] describes the class $\mathcal{A}\left(C^{\prime}\right)$, where $C^{\prime}=\mathbb{C} \backslash C$ stands for the complement of $C$.

Theorem 1.2. ([4] Let $T: \mathbb{C}[z] \rightarrow \mathbb{C}[z]$ be a linear operator. Then $T \in \mathcal{A}\left(C^{\prime}\right)$ if and only if, either

(1) The range of $T$ is of dimension 1 and $T$ can be written as

$$
T(f)=\alpha(f) P,
$$

where $\alpha$ is a linear functional, and $P \in \pi\left(C^{\prime}\right)$, or

(2) For any non-negative integer $n$ the polynomial

$$
T\left[((a z+b)(c w+d)+(a w+b)(c z+d))^{n}\right]
$$

is C-stable.

There is a similar theorem for operators acting on $\mathbb{C}_{n}[z]$, the space of all polynomial of degree at most $n$.

Theorem 1.3. ([4]) Let $T: \mathbb{C}_{n}[z] \rightarrow \mathbb{C}[z]$ be a linear operator. Then $T$ acts from $\pi_{n}\left(C^{\prime}\right)$ to $\pi\left(C^{\prime}\right) \cup\{0\}$ if and only if, either

(1) The range of $T$ is of dimension 1 and $T$ can be written as

$$
T(f)=\alpha(f) P,
$$

where $\alpha$ is a linear functional, and $P \in \pi\left(C^{\prime}\right)$, or

(2) The polynomial

$$
T\left[((a z+b)(c w+d)+(a w+b)(c z+d))^{n}\right]
$$

is C-stable. 
In [4 a similar description is also given for the class $\mathcal{A}(K)$ in the case where $K$ is a line or a circle. However, in the case where $K$ is an open circular domain the description of $\mathcal{A}(K)$ was formulated as an open problem in [4, 6] as well as in [3, Section 5], where conditions which are separately necessary or sufficient were obtained for an operator to preserve roots in the open half-plane.

The aim of this note is to give a complete description of operators preserving roots in an open circular domain which is similar to Theorem 1.2. It will be derived from Theorem 1.2 and from Theorem 2.4. describing the difference between the classes $\mathcal{A}(G)$ and $\mathcal{A}(\bar{G})$, where $G$ is an open set in $\mathbb{C}$. Theorem 2.4 is, in its turn, a corollary of the classical Hurwitz theorem.

\section{Difference between $\mathcal{A}(G)$ and $\mathcal{A}(\bar{G})$}

In this section $G$ is always an open set in the complex plane. First, let us show that $\mathcal{A}(G) \subset \mathcal{A}(\bar{G})$.

Proposition 2.1. Let $T: \mathbb{C}[z] \rightarrow \mathbb{C}[z]$ be a linear operator. If $T \in \mathcal{A}(G)$, then $T \in \mathcal{A}(\bar{G})$.

Proof. Assuming the opposite, we can find a polynomial $p \in \pi(\bar{G})$ such that $T p \notin$ $\pi(\bar{G})$. Then there exists a root $z_{0}$ of $p(z)$ lying on $\partial G$ (otherwise $T p \in \pi(G)$ ). Let $p(z)=\left(z-z_{0}\right) r(z)$. Consider a family of polynomials:

$$
p_{\zeta}(z)=p(z)-\zeta r(z)=\left(z-z_{0}-\zeta\right) r(z) .
$$

By continuity argument, there exists $\zeta$ such that $z_{0}+\zeta \in G$ and $T p_{\zeta} \notin \pi(\bar{G})$. Applying the similar reasoning for each root of $p(z)$, which belongs to $\partial G$, we will get $q \in \pi(G)$ such that $T q \notin \pi(G)$. We come to a contradiction with the condition $T \in \mathcal{A}(G)$.

However, $\mathcal{A}(G) \neq \mathcal{A}(\bar{G})$. It is easy to find an operator from $\mathcal{A}(\bar{G}) \backslash \mathcal{A}(G)$ (e.g., let $G$ be an open upper half-plane and let $(T p)(z)=z p(z))$.

Denote by $\mathbb{C}^{(n)}[z]$ the space of polynomials of degree exactly $n$, and denote by $\pi^{(n)}(K)$ the set of polynomials of degree exactly $n$ with all roots in the set $K$. For $\mathcal{P} \subset \mathbb{C}[z]$, denote by $G C D(\mathcal{P})$ the greatest common divisor of all polynomials from $\mathcal{P}$.

Lemma 2.2. Let $G \subset \mathbb{C}$ be an open set and $T \in \mathcal{A}(\bar{G})$. Then the following are equivalent:

(1) There exists $p \in \pi^{(n)}(G)$ such that $T p \notin \pi(G) \cup\{0\}$;

(2) $G C D\left(T\left(\pi^{(n)}(G)\right)\right)$ has a root on $\partial G$.

Proof. Implication $2 \Longrightarrow 1$ is obvious. Let us prove $1 \Longrightarrow 2$. Assuming the opposite, we can find two polynomials $p, q \in \pi^{(n)}(G)$ and $\xi \in \partial G$ such that $(T p)(\xi)=0$, but $(T q)(\xi) \neq 0$. Without loss of generality we may assume that $0 \in \partial G$ and $\xi=0$. Let $m$ be a multiplicity of zero as a root of $T(p)$. Therefore $T(p)(z)=z^{m} R(z)$. If we put $Q=T q$, then $Q(0) \neq 0$. Since $G$ is open, applying the Hurwitz theorem we can find $\varepsilon>0$ such that $p+\zeta q \in \pi^{(n)}(G)$ for all $\zeta$ with $|\zeta|<\varepsilon$. Thus, $T(p+\zeta q)=z^{m} R(z)+\zeta Q(z) \in \pi(\bar{G})$. Choose $z \notin \bar{G}$ with $|z|$ small enough so that

$$
|\zeta|=\left|-z^{m} \frac{R(z)}{Q(z)}\right|<\varepsilon
$$

Then $T(p+\zeta q)(z)=0$, which contradicts the assumption that $T(p+\zeta q) \in \pi(\bar{G})$. 
Actually, one can replace the second condition in the above lemma with a condition, which is much easier to check. We need a simple statement for that.

Lemma 2.3. Let $T: \pi_{n}(G) \rightarrow \pi(G) \cup\{0\}$ be a linear operator. If $n>k>m$, then

$$
G C D\left(T\left(\pi^{(m)}(G)\right)\right) \vdots G C D\left(T\left(\pi^{(k)}(G)\right)\right) .
$$

Proof. Without loss of generality we may take $m=k-1$. Let $a \in G$, one can find $\epsilon \in \mathbb{C}$ such that $a+\epsilon \in G$. For arbitrary $p \in \pi^{(k-1)}(G)$ we have

$$
T[(z-a-\epsilon) p(z)](\zeta)=[T((z-a) p)](\zeta)-\epsilon(T p)(\zeta) .
$$

Denote by $\zeta_{0}$ a root of $G C D\left(T\left(\pi^{(k)}(G)\right)\right)$. If we put $z=\zeta_{0}$ in the above equality, we get

$$
(T p)\left(\zeta_{0}\right)=0 .
$$

So, every root of $G C D\left(T\left(\pi^{(k)}(G)\right)\right)$ will also be a root of any polynomial from $T\left(\pi^{(k-1)}(G)\right)$ of at least the same multiplicity and, therefore,

$$
G C D\left(T\left(\pi^{(k-1)}(G)\right)\right) \vdots G C D\left(T\left(\pi^{(k)}(G)\right)\right) .
$$

Now we are ready to describe the difference between $\mathcal{A}(G)$ and $\mathcal{A}(\bar{G})$.

Theorem 2.4. Let $G \subset \mathbb{C}$ be an open set. Consider a linear operator $T: \mathbb{C}[z] \rightarrow$ $\mathbb{C}[z]$ preserving roots in $\bar{G}$. The following are equivalent:

(1) The operator $T$ belongs to $\mathcal{A}(G)$;

(2) If $k$ is a minimal non-negative integer such that $T\left[z^{k}\right]$ is not identically zero, then $T\left[z^{k}\right]$ has no roots on $\partial G$.

Proof. The second statement trivially follows from the first one. Let us prove that $1 \Longrightarrow 2$. Assuming the converse, we can find a polynomial $p \in \pi(G)$ such that $T p \notin \pi(G) \cup\{0\}$. Let $n$ be the degree of $p$. Applying Lemma 2.2. we get that $G C D\left(T\left(\pi^{(n)}(G)\right)\right)$ has a root on $\partial G$. Hence, by Lemma 2.3. $G C D\left(T\left(\pi^{(k)}(G)\right)\right)=$ $c T\left[z^{k}\right]$ has a root on $\partial G$. We arrive at a contradiction.

Remark. Let $T: \mathbb{C}[z] \rightarrow \mathbb{C}[z]$ be a linear operator. Consider its expansion as a series with respect to the differentiation operator $D$ :

$$
T=\sum_{k=0}^{\infty} Q_{k} D^{k}
$$

It is easy to see, that the second condition in Theorem 2.4 is equivalent to $Q_{k}(z) \neq 0$ for all $z \in \partial G$, where $Q_{k}$ is the first non-zero polynomial coefficient in the above expansion.

In conclusion we give an example of an operator $T \in \mathcal{A}(\bar{G}) \backslash \mathcal{A}(G)$ such that $G C D\left(\mathbb{C}_{n}[z]\right)$ has roots on $\partial G$ for some non-negative integer $n$, while $G C D\left(\mathbb{C}_{n+1}[z]\right)$ does not. Let $G$ be the upper half-plane $H$. Consider an operator $T$ given by

$$
(T p)(z)=a_{n+1}-a_{0} z,
$$

where $p(z)=\sum_{j=0}^{\infty} a_{j} z^{j}$ and only finitely many coefficients $a_{j}$ are non-zero. If all roots of $p$ are real, then the same is true for $T p$. So $T \in \mathcal{A}(\mathbb{R})$. It is known [4. Section 3.1, Lemma 3] that if $T \in \mathcal{A}(\mathbb{R})$, then it maps $\pi(\bar{H})$ either to $\pi(\bar{H})$ 
or to $\pi(-\bar{H})$. It is easy to see that $T(\pi(\bar{H})) \subset \pi(\bar{H})$, so $T \in \mathcal{A}(\bar{G})$. Finally, $G C D\left(\mathbb{C}_{n}[z]\right)=z$ has a root on $\mathbb{R}$, while $G C D\left(\mathbb{C}_{n+1}[z]\right)=1$ does not.

\section{Classification of linear operators PRESERving Roots in OPEN} CIRCULAR DOMAIN

In this section we will derive from Theorems 1.2 and 2.4 a description of linear operators preserving roots in open circular domain. As in Section $1, \Phi(z)=\frac{a z+b}{c z+d}$ and $C=\Phi^{-1}(H)$, where $H$ is the open upper half-plane $\{z: \Im z>0\}$. Denote by $C^{r}$ the set $\operatorname{Int}\left(C^{\prime}\right)$, that is, the interior of the complement $C^{\prime}$ of $C$.

For the sake of brevity, put

$$
Q_{n}(z, w)=((a z+b)(c w+d)+(a w+b)(c z+d))^{n} .
$$

The next theorem is an analogue of Theorem 1.2 for open circular domains.

Theorem 3.1. Let $T: \mathbb{C}[z] \rightarrow \mathbb{C}[z]$ be a linear operator. Then $T \in \mathcal{A}\left(C^{r}\right)$ if and only if either

(1) The range of $T$ is of dimension 1 and $T$ can be written as

$$
T(f)=\alpha(f) P,
$$

where $\alpha$ is a linear functional and $P \in \pi\left(C^{\prime}\right)$, or

(2) For any non-negative integer $n$ such that the polynomial $T\left[Q_{n}(z, w)\right]$ is not identically zero, $\left(T Q_{n}\right)(z, w) \neq 0$ whenever $z \in \bar{C}$ and $w \in C$.

Proof. Case (1) is trivial, so let us assume, that the range of $T$ has dimension greater than 1. First we prove the necessity of (2). Consider the equation

$$
(a z+b)(c w+d)+(a w+b)(c z+d)=0,
$$

which is equivalent to

$$
\frac{a z+b}{c z+d}=-\frac{a w+b}{c w+d} .
$$

If $w \in C$ and $z \in \bar{C}$, then

$$
\frac{a z+b}{c z+d}=\Phi(z) \in \bar{H},
$$

and, therefore,

$$
-\frac{a w+b}{c w+d} \in \bar{H} .
$$

Thus, $w \in C^{\prime}$, but this contradicts the assumption $w \in C$. So we have shown that $Q_{n}(z, w) \neq 0$ if $z \in \bar{C}$ and $w \in C$. Hence, for any fixed $w_{0} \in C$, the univariate polynomials $Q_{n}\left(z, w_{0}\right)$ belong to $\pi\left(C^{r}\right)$. Since $T \in \mathcal{A}\left(C^{r}\right)$, the same is true for the polynomials $T\left[Q_{n}\left(z, w_{0}\right)\right]$. Therefore $\left(T Q_{n}\right)(z, w) \neq 0$, if $z \in \bar{C}$ and $w \in C$.

We turn to the proof of sufficiency. It follows from condition (2) that the polynomials $T\left[Q_{n}(z, w)\right]$ are $C$-stable. Hence, by Theorem[1.2, $T \in \mathcal{A}\left(C^{\prime}\right)$. Let $k$ be the minimal non-negative integer such that $T\left[z^{k}\right] \neq 0$. By Theorem 2.4 , if $T \notin \mathcal{A}\left(C^{r}\right)$ then $T\left[z^{k}\right]$ should have roots on $\partial C^{r}=\partial C$. Therefore, $\left(T Q_{k}\right)(z, w)=\alpha(w) T\left[z^{k}\right]$ must vanish for some $z \in \bar{C}$ and $w \in C$. We arrive at a contradiction.

Remark. Condition (2) in Theorem 3.1 may be replaced by a slightly weaker condition. Let $k$ be the minimal non-negative integer such that $T\left[z^{k}\right]$ is not identically zero. Then condition (2) holds if and only if all polynomials $T\left[Q_{n}(z, w)\right], n>0$, are $C$-stable, and $\left(T Q_{k}\right)(z, w) \neq 0$ for $z \in \bar{C}$ and $w \in C$. This can be seen by applying the same reasoning as in the proof of Theorem 2.4 
However, one can not obtain similar classification of operators which act on the space $\mathbb{C}_{n}[z]$ and preserve roots in an open circular domain. The following statement could be an analogue of Theorem 1.3 for open circular domains:

$"$ Let $T: \mathbb{C}_{n}[z] \rightarrow \mathbb{C}[z]$ be a linear operator. Then $T\left(\pi_{n}\left(C^{\prime}\right)\right) \subset \pi\left(C^{\prime}\right)$ if and only if either

(1) The range of $T$ is of dimension 1 and $T$ can be written as:

$$
T(f)=\alpha(f) P,
$$

where $\alpha$ is a linear functional and $P \in \pi\left(C^{\prime}\right)$, or

(2) $\left(T Q_{n}\right)(z, w) \neq 0$ whenever $z \in \bar{C}$ and $w \in C$."

We show that this statement is not correct; namely, condition (2) turns out to be not sufficient. Consider the operator $(T p)(z)=p^{\prime}(z)-z p(z)$. Let $L$ be the lower half-plane $\{z: \Im z<0\}$, in this case $Q_{n}(z, w)=(z+w)^{n}$. First note that $T[1]=z$ and so $T \notin \mathcal{A}(L)$. However, by Theorem 1.2 and below reasoning $T \in \mathcal{A}(\bar{L})$. Let us evaluate $T\left[(z+w)^{n}\right]$ :

$$
T\left[(z+w)^{n}\right]=n(z+w)^{n-1}-z(z+w)^{n}=(z+w)^{n-1}[n-z(z+w)] .
$$

Suppose that $n>0$. Let us see that the obtained polynomials do not vanish when $z \in \bar{H}$ and $w \in H$. Since $(z+w)^{n-1} \neq 0$ for $z \in \bar{H}$ and $w \in H$, we look at the polynomial $q(z, w)=n-z(z+w)$. For $z \in \bar{H}$ and $w \in H$, we have $0 \leq \arg z \leq \pi$, $0<\arg w<\pi$, whence $0 \leq \arg (z+w) \leq \pi$ and $0<\arg [z(z+w)]<2 \pi$. Clearly, in this case $q(z, w) \neq 0$. So, if $n>0$, then $T\left[(z+w)^{n}\right] \neq 0$ for $z \in \bar{H}$ and $w \in H$. Thus, condition (2) is fulfilled but it is not sufficient for the inclusion $T\left(\pi_{n}(L)\right) \subset \pi(L)$.

\section{ACKNOWLEDGEMENTS}

The author wishes to thank Anton Baranov for numerous advises and discussions. The author is also grateful to Petter Brändén for useful remarks, especially for pointing out an inaccuracy in the initial version of Lemma 2.2

\section{REFERENCES}

[1] A. Aleman, D. Beliaev, H. Hedenmalm, Real zero polynomials and Pólya-Schur type theorems, J. Anal. Math. 94 (2004), 49-60.

[2] E. W. Benz, Über lineare verschiebungstreue Funktionaloperatoren und die Nullstellen ganzer Funktionen, Comment. Math. Helv. 7 (1934), 243-289.

[3] J. Borcea, P. Brändén, Multivariate Pólya-Schur classification problems in the Weyl algebra, Proc. Lond. Math. Soc. (3) 101 (2010), 1, 73-104.

[4] J. Borcea, P. Brändén, Pólya-Schur master theorems for circular domains and their boundaries, Ann. of Math. (2) 170 (2009), 1, 465-492.

[5] G. Pólya, I. Schur, Über zwei Arten von Faktorenfolgen in der Theorie der algebraischen Gleichungen, J. Reine Angew. Math. 144 (1914), 89-113.

[6] J. Borcea, P. Brändén, G. Csordas, V. Vinnikov, Report on the workshop "Pólya-Schur-Lax problems: hyperbolicity and stability preservers" (American Institute of Mathematics, Palo Alto, California, May 28 2007-June 1, 2007), http://www.aimath.org/pastworkshops/polyaschurlaxrep.pdf

Department of Mathematics and Mechanics, St. Petersburg State University, 28, Universitetskii Pr., St. Petersburg, 198504, Russia

E-mail address: eugeny.melamud@comapping.com 